\title{
Extreme Light Infrastructure: architecture and major challenges
}

\author{
J-P. Chambaret ${ }^{1}$, O. Chekhlov², G. Chériaux ${ }^{3}$, J. Collier ${ }^{2}$, R. Dabu ${ }^{4}$, P. Dombi ${ }^{5}$, A.M. Dunne ${ }^{2}$, \\ K. Ertel ${ }^{2}$, P. Georges ${ }^{6}$, J. Hebling ${ }^{7}$, J. Hein ${ }^{8}$, C. Hernandez-Gomez ${ }^{2}$, C. Hooker ${ }^{2}$, S. Karsch ${ }^{9}, 10$, \\ G. Korn', F. Krausz ${ }^{9}, 10$, C. Le Blanc ${ }^{11}$, Zs. Major ${ }^{9}, 10$, F. Mathieu ${ }^{1}$, T. Metzger ${ }^{9}$, G. Mourou ${ }^{1}$, \\ P. Nickles ${ }^{12}$, K. Osvay ${ }^{*}{ }^{1,13}$, B. Rus ${ }^{14}$, W. Sandner ${ }^{12}$, G. Szabó ${ }^{13}$, D. Ursescu ${ }^{4}$, K. Varjú ${ }^{13}$ \\ ${ }^{1}$ Institute de Lumiére Extreme, ENSTA, CNRS, Chemin de la Huniere, Palaiseau Cedex, France \\ ${ }^{2}$ Central Laser Facility, Ruherford Appleton Laboratory, Chilton, Oxon, U.K. \\ ${ }^{3}$ LOA, ENSTA, CNRS, Chemin de la Huniere, Palaiseau Cedex, France \\ ${ }^{4}$ National Institute for Lasers, Plasma and Radiation Physics, Magurele, Romania \\ ${ }^{5}$ Research Institute for Solid State Physics and Optics, Budapest, Hungary \\ ${ }^{6}$ Institut d'Optique, Campus de Polytechnique, RD12, Palaiseau Cedex, France \\ ${ }^{7}$ Institute of Physics, University of Pecs, Hungary \\ ${ }^{8}$ Institute of Optics and Quantum Electronics, Friedrich-Schiller Universität, Jena, Germany \\ ${ }^{9}$ Max-Planck Institut für Quantenoptik, Garching, Germany \\ ${ }^{10}$ Ludwig-Maximilians-Universität, München, Germany \\ ${ }^{11}$ LULI, Ecole Polytechnique, Route de Saclay, Palaiseau, France \\ ${ }^{12}$ Max-Born-Institut, Berlin, Germany \\ ${ }^{13}$ Department of Optics \& Quantum Electronics, University of Szeged, Hungary \\ ${ }^{14}$ PALS, Institute of Physics, Prague, Czech Republic
}

\begin{abstract}
Extreme Light Infrastructure (ELI), the first research facility hosting an exawatt class laser will be built with a joint international effort and form an integrated infrastructure comprised at last three branches: Attosecond Science (in Szeged, Hungary) designed to make temporal investigation at the attosecond scale of electron dynamics in atoms, molecules, plasmas and solids. High Field Science will be mainly focused on producing ultra intense and ultra short sources of electons, protons and ions, coherent and high energetic X rays (in Prague, Czech Republic) as well as laserbased nuclear physics (in Magurele, Romania). The location of the fourth pillar devoted to Extreme Field Science, which will explore laser-matter interaction up to the non linear QED limit including the investigation of vacuum structure and pair creation, will be decided after 2012. The research activities will be based on an incremental development of the light sources starting from the current high intensity lasers (APOLLON, GEMINI, Vulcan and PFS) as prototypes to achieve unprecedented peak power performance, from tens of petawatt up to a fraction of exawatt $\left(10^{18} \mathrm{~W}\right)$. This last step will depend on the laser technology development in the above three sites as well as in current high intensity laser facilities.
\end{abstract}

Keywords: ELI, high intensity lasers, diode pumped solid state laser (DPSSL), optical parametric chirped pulse amplification (OPCPA), atto-physics, extreme field physics, photonuclear physics

\section{INTRODUCTION}

The Extreme Light Infrastructure (ELI) project was selected in 2006 by the European Strategic Forum for Research Infrastructure (ESFRI) committee as one of the 37 major research infrastructure projects. ELI now is about to complete its Preparatory Phase (ELI-PP) funded by the EC, in which 13 EU countries and around 50 academic institutions have been involved [1]. The site selection process of ELI-PP has concluded that the ELI Research Infrastructure would be

\footnotetext{
*karoly.osvay@ensta.fr, osvay@physx.u-szeged.hu
} 
implemented in three sites under a single governance: the Attosecond Science Facility in Szeged, Hungary, the High Field Science in Prague, Czech Republic, while the Nuclear Physics Science in Magurele, Romania. The location of the Extreme Field Science will be decided after 2012.

The primary objective of ELI project is to provide the worldwide scientific community a unique laser based research infrastucture, allowing them to investigate an unexplored domain of laser-matter interaction at the highest intensity level ever achieved [2-3], of the order reaching up to $10^{25} \mathrm{Wcm}^{-2}$, which is more than three orders of magnitude higher than today's state of the art. ELI will also be unique as it will produce a very broad range of ultrashort secondary sources devoted to various applications in physics, chemsitry and biomedicine, including high energy photons, electrons, protons, neutrons, and muons, in the attosecond and possibly zeptosecond regimes, $\mathrm{THz}$ and $\mathrm{X}$-ray, as well as gammaray beams. The scientific activities in the future ELI are focused to three main areas. Attosecond science aims at conducting attosecond scale time-domain studies of electron dynamics in atoms, molecules, plasmas and solids. High intensity beamlines are dedicated for generation of ultra short pulses of high energy radiation and particles up to tens of $\mathrm{GeV}$ for users. Finally, extreme field science will explore laser matter interaction up to the nonlinear QED limit including the investigation of pair creation and vacuum structure.

Separated but synchronised laser branches are designed to fulfill the scientific mission of ELI. Each of them challenges the state of the art technology at several points. Commonly, the repetition rate of the machines are planned at least one order of magnitude higher than in the currnetly running projects. In addition, the energy to be delivered to the target is typically a few times higher, as well. The far largest issue is to deliver the peak intensity pulses of 200PW. Before starting the design of this exawatt machine, the major technical and scientific bottlenecks have to be overcome first, in co-operation with the national laser projects and also in synergy with the planned fusion energy laser facility HiPER [4].

On of the main bottlenecks of the increase the repetition rate of high intensity lasers is the lack of suitable pump lasers. Due to heat management problems, the $100 \mathrm{~J}$ level flashlamp based technology does not seem suitable to increase the repetition rate above $0.1 \mathrm{~Hz}$. There are several national projects running in Europe to develop high repetition rate diode pumped solid state laser (DPSSL) as for pump sources. The approach of MBI in Berlin and MPQ in Garching are based on thin disk technology, and aiming to reach few $\mathrm{J}$ energy level pulses at $100 \mathrm{~Hz}$ and $1 \mathrm{kHz}$ repetition rate, respectively. Within the national project DiPOLE in the UK, a multiple slab technology is developed to reach the $\mathrm{kJ}$ level at a modest, but still remarkable repetition rate of $1 \mathrm{~Hz}$. Besides, there are further high intensity laser projects in Europe which are based on diode laser technology, as LUCIA in France and POLARIS in Jena.

Current ultrashort pulse, high intensity laser projects in Europe are utilizing different approaches. Three of them are of the highest importance for ELI, as they can be regarded forerunner machines. Two of them, the APOLLON project in France and VULCAN in the UK are aiming at the same peak power level of 10PW and similar repetition rate of $<0.05 \mathrm{~Hz}$, but they are utilizing different laser technologies. While the gain medium in the final amplifier of APOLLON is Ti:S, the VULCAN solution is the full optical parametric chirped pulse amplification (OPCPA). In both cases the pump sources of the final amplifiers are flashlamp-pumped Nd:Glass lasers. The Petawatt Field Syntheser (PFS) in Germany aims at generation of energetic attosecond pulses, so the peak power of the pulses are at PW level, but the aimed repetition rate is $10 \mathrm{~Hz}$. The pump source cannot be anything else but DPSSL. All the three projects are to be completed between 2011-2014.

In the following sectons first the national prototype projects are briefly described which are having especial importance toward the design and built of the ELI maschines. At last, the current, but far not finalized layout of the three ELI machines are described.

\section{NATIONAL PROTOTYPES}

\subsection{Apollon at Institute Lumiere Extreme (ILE), France}

The French "single beamline" laser prototype, APOLLON, is presently under construction with the purpose of addressing all issues related to the flashlamp-pumped "Titanium Sapphire" approach. We will present here the developments under investigation to reach the expected specifications of laser pulses with $150 \mathrm{~J} / 15 \mathrm{fs}(10 \mathrm{PW})$ at a repetition rate of at least 1 shot per minute. The intensity on target is expected $i$ in the range of $10^{23}$ to $10^{24} \mathrm{~W} / \mathrm{cm}^{2}$. 


\subsubsection{Overview}

The scheme of APOLLON is shown on Figure 1. To seed the power amplifiers we need a well adapted Front End part delivering at high repetition rate Ultrashort pulses (5 to $10 \mathrm{fs}$ ) and with a very large bandwidth necessary to compensate the gain narrowing occurring through the power amplification process. For that we have developed an OPCPA front pumped by thin disk diode pumped lasers at $100 \mathrm{HZ}$, developed jointly with the Max Born Institute, Berlin, Germany.

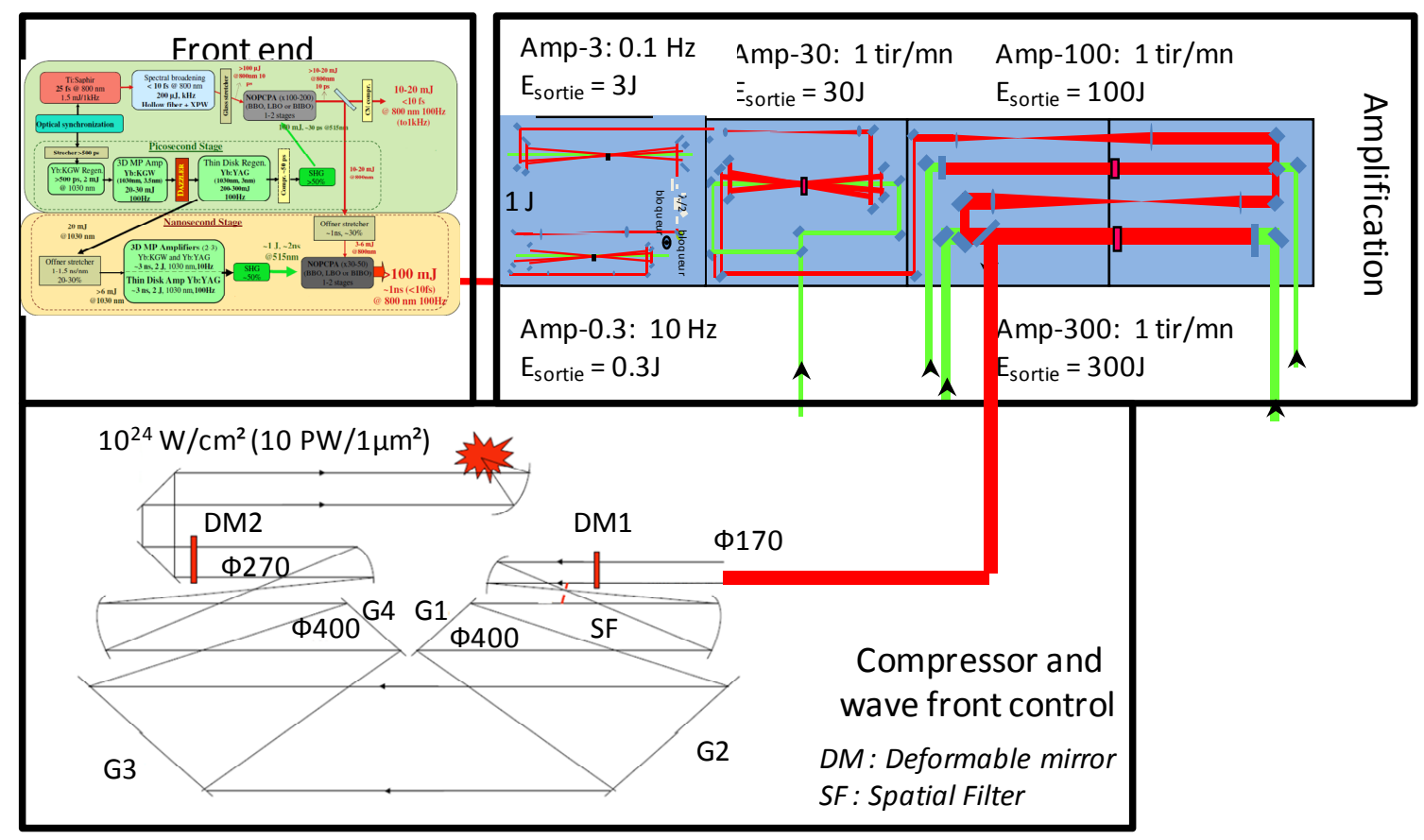

Figure 1. Schematics of the Apollon laser.

Pump laser must deliver on these crystals energy in the green as high as 600 to $800 \mathrm{~J}$ in nanosecond range, with a reasonable number of laser units avoiding the use of hundred of NdYag lasers delivering each few Joules at 532nm. The development of new lasers systems delivering hundreds of Joules in the second harmonic must be studied, with the highest achievable repetition rates. Our lower limit was fixed at 1 shot per minute. To optimize the performances of such lasers, SHG conversion must be adapted to limit the required infrared energy, and the associated thermal load in the pump laser amplifiers. For that we are studying the availability of large size LBO crystals, which are very efficient to convert the infrared laser pulses, exhibit a high damage threshold, and a large angular acceptance.

Moreover, the pump fluency on the large TiSa is close to the damage threshold of the material and requires a good control of the spatial energy distribution to avoid hot spots which could damage these expensive crystals. For smoothing these pump energy distribution we are developing new homogenizers based on Diffractive Optical Elements allowing to prevent the pumped crystal from any change in the pump energy distribution.

\subsubsection{Scientific and technical bottlenecks}

The weakest optical element in the Ultra intense CPA laser systems has always been the compression gratings. Those gratings must exhibit a broad spectral diffraction efficiency $(200 \mathrm{~nm}$ centered around $800 \mathrm{~nm}$ ) allowing recompression of pulses as short as 10- 15fs without spectral clipping effects. Only gold gratings could be used today for that reason, but these gratings present low damage threshold (below $200 \mathrm{~mJ} / \mathrm{cm}^{2}$ ), leading to the use of large and expensive off axis parabolic mirrors based beam expanders to extend the input beam to $40 \mathrm{~cm}$ in diameter. This huge beam diameter induces the need of extremely large gratings (on the meter size range). To overcome this problem we are presently investigating new schemes of diffraction gratings based on combination of metallic and dielectric layers we will present below in detail.

A main issue consists in the spatial quality of the beam and for that purpose we are working on developing new deformable mirrors with a broadband hard coating $(700-900 \mathrm{~nm})$ and with diameters as high as $200 \mathrm{~mm}$. We plane to 
associated such Deformable Mirrors (DM) to high damage threshold grazing incidence dielectric spatial filters in order to remove high spatial frequencies wavefront distortions susceptible to generate hot spots on the compression gratings.

Finally we are also investigating new High Reflective, Broadband, High Damage Threshold mirror coatings allowing propagating hundreds of Joules of 10 - 15 fs pulses under vacuum from compressor to the experimental chambers.

\subsection{Astra-Gemini at Central Laser Facility, RAL, U.K.}

\subsubsection{Overview}

Astra Gemini is an upgrade of the existing Astra Ti:Sapphire (Ti:S) laser system. The general layout is illustrated in Fig. 2(a): the pulse from Astra with an energy of $1.5 \mathrm{~J}$ is split in two and each pulse is amplified in a separate four-pass Ti:S amplifier to an energy of $25 \mathrm{~J}$ and then compressed in a separate grating compressor. Each amplifier is pumped by up to $62 \mathrm{~J}$ of green light from a Nd:glass laser system operating at up to one shot per $20 \mathrm{~s}$. The two re-compressed pulses ( $30 \mathrm{fs}, 15 \mathrm{~J}$, equiv. $0.5 \mathrm{PW}$ ) are delivered to an interaction chamber that is housed in a concrete bunker located underneath the laser room. This bunker with $1 \mathrm{~m}$ thick walls provides both radiation shielding and a very stable platform for the laser system, as shown in Fig. 2(b). With the chosen set-up, the two beams are synchronised, yet individually configurable in terms of energy, pulse duration and relative delay. This will enable a wide range of plasma physics experiments, e.g. pump-probe and backlighter configurations and the combination of a laser beam with a laser-generated particle or x-ray beam.

(a)

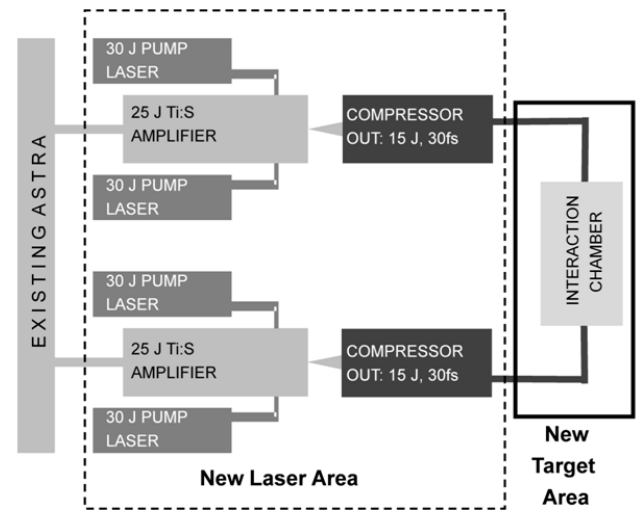

(b)

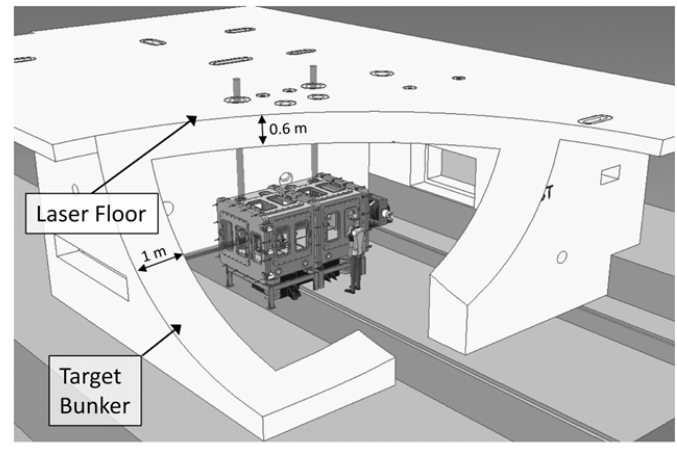

Figure 2. Schematic layout of Astra Gemini (a) and building layout (b).

\subsubsection{Beam transport}

Great care has been taken to preserve a good beam quality by relay-imaging the beam all the way from the output of Astra to the grating compressors. This path is about $80 \mathrm{~m}$ long and contains six vacuum relay telescopes, three of which also act as beam expanders and the other three as spatial filters. Where necessary, the telescopes are chromatically corrected to avoid pulse front distortions [5]. We have devised a novel design for the final telescope that expands the beam from $50 \mathrm{~mm}$ to $150 \mathrm{~mm}$. It contains a fused silica singlet output lens and a special doublet input lens that precompensates the chromatic error of the output lens, making the telescope achromatic as a whole. More detailed information can be found in [6].

\subsubsection{Pulse amplification and compression}

The two amplifiers each contain a $90 \mathrm{~mm}$ diameter Ti:S crystal, with the diameter of the pump end extraction beams being $50 \mathrm{~mm}$. The extraction beam passes the crystal four times under different angles. The beam is spatially filtered after each of the first three passes. The crystal is pumped from both sides with up to $30 \mathrm{~J}$ each by pulses from a frequency doubled Nd:glass laser system (Quantel SA). The largest amplifier rods in the pump laser are $45 \mathrm{~mm}$ in diameter. Due to the high repetition rate of 1 shot per $20 \mathrm{~s}$, the thermal stress in these rods is considerable, leading to a poor beam profile, as shown in Fig. 3(a). We have overcome this problem by employing diffractive optical elements (DOEs) for beam homogenisation. These DOEs (Silios Technologies) provide both a very uniform pump profile, as shown in Fig. 3(b) and very high diffraction efficiencies in excess of $93 \%$. 
Another serious challenge related to large aperture Ti:S amplifiers is amplified spontaneous emission (ASE) because of the very high gain-length product that is experienced by photons travelling perpendicular to the optical axis. To minimise ASE-related losses it is necessary to minimise this transverse gain and to suppress Fresnel reflections at the crystal surface in order to prevent parasitic lasing. We have minimised the transverse gain by choosing a weakly doped Ti:S crystal and double-passing the pump beams through the crystal. The suppression of Fresnel reflections at the cylindrical face of the crystal and hence of parasitic lasing is provided by an index-matched absorber liquid. Detailed information on pump beam homogenisation, double-pass pumping and parasitic lasing suppression can be found in [7]. With pump homogenisation and ASE suppression in place, $26 \mathrm{~J}$ of amplified pulse energy were obtained with $62 \mathrm{~J}$ of pump energy.


Figure 3. Pump beam profiles before (a) and after homogenisation (b).

After amplification, the beam is expanded to $150 \mathrm{~mm}$ diameter and pulses are recompressed in a vacuum compressor containing two large size gold-coated diffraction gratings in a double pass configuration. The shortest pulse duration measured so far was $40 \mathrm{fs}$.

\subsubsection{First Plasma Physics Experiments}

Since its commissioning, the Astra Gemini facility has played host to numerous user groups investigating a variety of high intensity laser plasma interactions, using both solid and gaseous targets. One highlight so far has been the acceleration of electrons in a free-space gas jet to nearly $1 \mathrm{GeV}[8]$.

\subsection{Petawatt Field Synthesizer at MPQ Garching, Germany}

The Petawatt Field Synthesizer (PFS) project at the Max-Planck-Institut für Quantenoptik (MPQ) was started in 2005 in order to provide phase-stable few-cycle pulses at petawatt-class peak powers and high repetition rates for high-efficiency single attosecond pulse generation from solid surfaces and stable high-charge electron beams via bubble acceleration. The envisioned pulse parameters are 5 fs duration, $3 \mathrm{~J}$ energy and $10 \mathrm{~Hz}$ repetition rate.

\subsubsection{General design principles}

Presently the only realistic way of generating few-cycle pulses at energies above a few $\mathrm{mJ}$ seems to be non-collinear optical-parametric amplification (NOPA) using a three-wave mixing process inside a nonlinear crystal. Here, the amplification bandwidth is determined by the phase-matching properties and length of the chosen crystal. Broadband crystals such as BBO or LBO are still not readily available in sizes sufficient for petawatt peak powers. Therefore a modified approach was chosen for PFS, which offers several additional benefits and is schematically outlined in Fig. 4.

The main design idea is the use of short (few-mm) DKDP crystals for NOPA amplification, which in turn require high pump intensities in order to achieve sufficient gain over their short length. This necessitates the use of picosecond, high energy pump pulses, which are provided by a specially designed diode-pumped Yb:YAG CPA laser with a design output of $4 \times 10 \mathrm{~J}$ at $1030 \mathrm{~nm}$ and $1 \mathrm{ps}$ duration. Since there is no pump light outside this $1 \mathrm{ps}$ temporal window, PFS is expected to reach an inherently high temporal contrast. The downside of this approach is the necessity for sub-100 fs 
synchronization between the pump pulses and the signal beam, which can only be achieved by an optical synchronization scheme. Since the pump light absorption in the NOPA crystals is almost negligible, it is expected that the PFS scheme can be scaled to high repetition rates for ELI by employing a suitable pump laser. Currently the PFS is in an advanced state of construction, and more details can be found in [9-13].


Figure 4. (left) PFS design philosophy, explaining the use of thin DKDP crystals for ps-pumped NOPA amplification driven by a diode-pumped Yb:YAG CPA laser. (right) schematic layout of PFS, detailing the frontend, pump-laser and NOPA-chain functional units.

\subsubsection{Front end}

The frontend provides the seed pulses for the pump laser and the NOPA chain in their respective spectral regions with few-10 fs synchronization. They are both derived for the output of an ultrabroadband, phase stabilized Ti:Sapphire oscillator. The seed pulses for the pump laser are split off and spectrally shifted to $1030 \mathrm{~nm}$ directly at the oscillator output, while the remainder is post-amplified to $2 \mathrm{~mJ}$ and compressed to $19 \mathrm{fs}$ in a commercial Femtopower Ti:Sapphire amplifier. The pulses are spectrally broadened in a two-stage hollow-core fiber setup to yield a continuum ranging from $250 \mathrm{~nm}$ to $1400 \mathrm{~nm}$. Its spectral phase was measured to be sufficiently smooth to be compensated by a combination of chirped mirrors and a Dazzler. These pulses are used as a seed for the demonstration of the first broadband NOPA stage, which will be described below.

\subsubsection{Pump laser}

The $1030 \mathrm{~nm}$ frequency-shifted output from the oscillator is fed into a two-stage Yb:glass fiber amplifier built by the Institute for Applied Physics (IAP) in Jena, which at its output provides $14 \mathrm{~nJ}$ pulses in a $10 \mathrm{~nm}$ spectral range. They are further stretched to $2 \mathrm{~ns}$ in a grating stretcher accepting a $3.5 \mathrm{~nm}$ spectral window, and subsequently pass through a second- zero dispersion stretcher with a spatial light modulator (SLM) in order to pre-compensate for spectral narrowing in the main Yb:YAG amplifiers. Without such spectral shaping gain narrowing in the amplification stages following the stretcher would lead to a final bandwidth of $<1 \mathrm{~nm}$ and thus require impractical dispersion values (i.e. grating separations) in the stretcher and compressor in order to maintain a safe, several-ns pulse duration during amplification. The SLM setup will be replaced by a Dazzler in the near future for more flexibility and higher throughput. After the stretcher the shaped pulses are amplified by approx. 100 passes in a $10 \mathrm{~Hz}$, diode-pumped Yb:glass regenerative amplifier (regen) to $200 \mu \mathrm{J}$ without significant spectral modification. The regen is followed by the first diode pumped Yb:YAG 8-pass amplifier for a boost to $300 \mathrm{~mJ}$ energy at $10 \mathrm{~Hz}$, keeping the full $3.5 \mathrm{~nm}$ bandwidth. At this stage, the pulses are currently passed into the compressor using multilayer-dielectric gratings with $6.5 \mathrm{~m}$ grating separation and compressed down to $1 \mathrm{ps}$ duration with $200 \mathrm{~mJ}$ pulse energy. These pulses are frequency doubled to $515 \mathrm{~nm}$ using a DKDP crystal, currently providing up to $80 \mathrm{~mJ}, 1$ ps pulses for the NOPA development.

In parallel to this, the development and optimization of the remaining Yb:YAG stages is ongoing. After having demonstrated up to 3J pulses from an Yb:YAG amplifier in slab geometry [10] at $1 \mathrm{~Hz}$ repetition rate, it became clear that a thick-disk approach will be better suited for reaching the repetition rate and efficiency goals of $10 \mathrm{~Hz}$ at PFS, since it will allow a multi-pass pumping scheme [14] and a more effective ratio of heat extraction surface and laser beam crossection. Therefore, both a new $500 \mathrm{~mJ}$ (instead of $300 \mathrm{~mJ}$ ) amplifier and a subsequent $2 \mathrm{~J}$ stage are under 
construction, which have already demonstrated up to $1 \mathrm{~J}$ in a strongly simplified test setup. Depending on the experience with this novel design, the $10 \mathrm{~J}$ stages will be scaled up from this approach.

\subsubsection{NOPA chain}

The seed pulses for the NOPA chain are cut from the 250-1400 nm continuum delivered by the frontend and are stretched using a double prism pair setup to about $1.3 \mathrm{ps}$ pulse duration. These pulses have a negative chirp and a spectral range of 700-1400 nm.

Before well-synchronized pump and seed pulses from PFS itself were available, short-pulse NOPA test experiments have been successfully conducted using a high-energy Ti:sapphire laser to investigate various critical aspects of shortpulse NOPA such as the verification of the predicted bandwidth and mode quality, and the necessity for pulse-front matching when two short laser pulses interact in the NOPA crystal at a non-zero angle [15]. These experiments confirmed the principal feasibility of scaling short-pulse NOPA to large beam diameters and confirmed the validity of the theoretical calculations.

Very recently, synchronized pump and seed pulses from PFS became available and preliminary OPCPA experiments confirm the predicted amplification bandwidth (see Fig. 5). The detailed results will be discussed elsewhere.

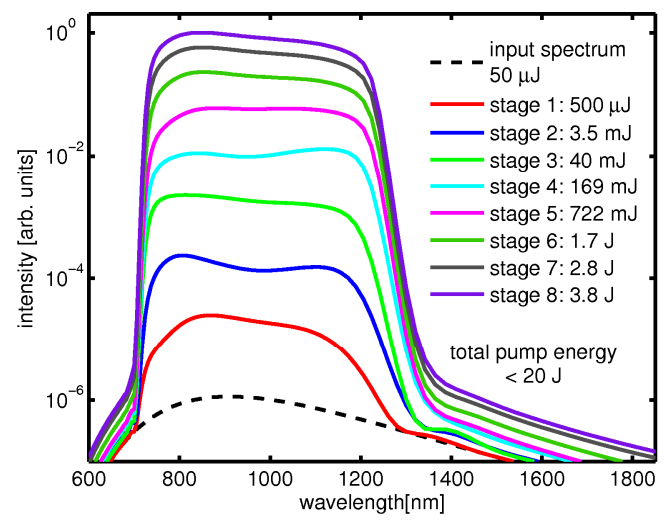

Figure 5. Left: 1-dimensional design calculations for the PFS optical parametric amplification chain. The calculations shows that amplification to the Joule-level is possible in DKDP, while a sufficiently broad spectrum for a fewcycle pulse is also supported.

\subsection{VULCAN 10PW at Central Laser Facility, RAL, U.K.}

The Vulcan 10 PW project aims to upgrade the Vulcan laser, located at the United Kingdoms' Central Laser Facility (CLF) to beyond the $10 \mathrm{PW}$ power level and provide focussed intensities of greater than $10^{23} \mathrm{~W} / \mathrm{cm}^{2}$ to its UK and international user community. This will be achieved by generating pulses with energies of $300 \mathrm{~J}$ and with durations less than 30fs. The upgrade will rely on OPCPA [16]. It has already been the subject of a great deal research over the last decade, including at the CLF [17-20].

\subsubsection{Design principles}

The design is based on a triple OPCPA amplification scheme with initial amplification occurring in the picosecond domain and subsequent amplification occurring in the nanosecond domain to guarantee a high contrast pulse. The project was divided into two phases, with phase one now completed. Phase two will commence in April 2010. A novel Front End has been developed during phase one to seed the remainder of the laser system [21]. This Front End comprises of a milli-Joule level broadband seed, a stretcher and an OPCPA Joule level amplifier based on LBO crystals. It delivers ultra-short pulses at $910 \mathrm{~nm}$, with energies of up to 1 Joule and with a bandwidth of $>150 \mathrm{~nm}$, sufficient to support a pulse duration of $30 \mathrm{fs}$ or less.

The second phase will produce both a high energy amplified pulse and the associated compression / target interaction equipment to enable user experiments. The Joule level output from the Front End system will enter a final OPCPA system, based on two large aperture (DKDP) crystals, for amplification to the $500 \mathrm{~J}$ level. These two crystals will be 
pumped by $\sim 3$ ns duration beams from Vulcan. To achieve this level of energy two Vulcan high energy beam lines will be upgraded through the installation of additional $\mathrm{Nd}$ : glass $208 \mathrm{~mm}$ diameter amplifier chains. The amplified output beam will then be compressed in time and directed to one of two different interaction areas generating focussed intensities of greater than $10^{23} \mathrm{~W} / \mathrm{cm}^{2}$. In the first area, the $10 \mathrm{PW}$ beam will be directed to the existing Target Area Petawatt (TAP) to be combined with the existing $1 \mathrm{PW}$ beam from Vulcan in a 1+10 PW orthogonal configuration. In addition, the existing TAP area will been extended to enable a class of experiments that require the use of a long F\#20 focussing optic. The $10 \mathrm{PW}$ beam will also be directed to a new heavily shielded interaction area called the High Intensity Area (HIA) and with the aid of an F\#0.7 focussing optic intensities $10^{23} \mathrm{~W} / \mathrm{cm}^{2}$ will be reached. A significant multi-floor extension to the existing Vulcan building will be required, the ground floor of which is shown in Fig. 6 .

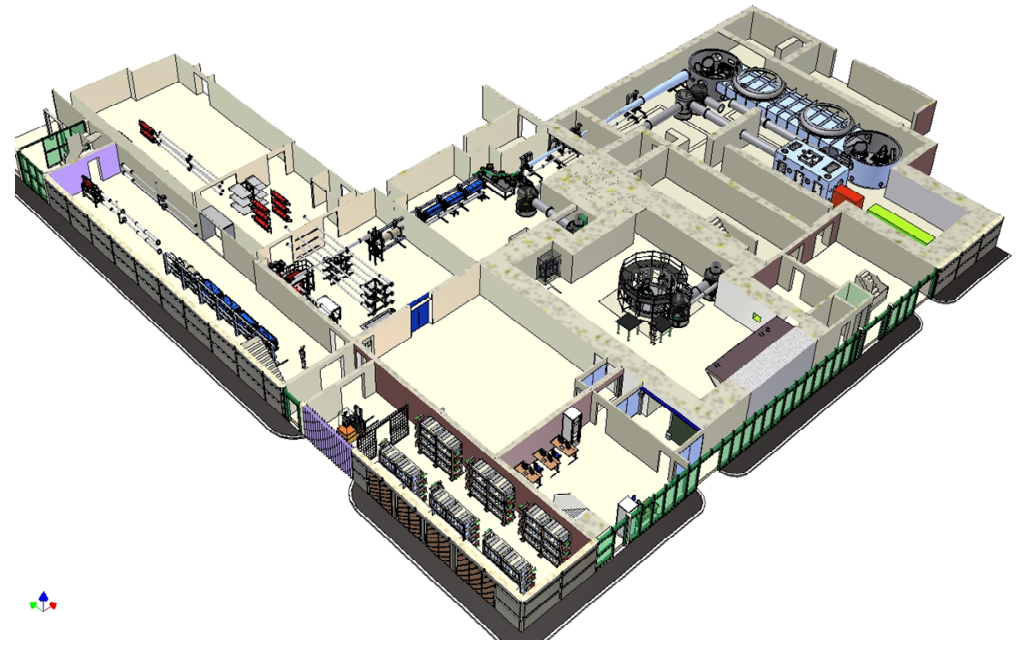

Figure 6. View of the ground floor of the building reconstructed for VULCAN 10 PW.

\subsubsection{Diode pumped cryogenic kJ pump laser for VULCAN (DiPOLE)}

Developing kJ-class diode pumped solid state lasers with multi-Hz repetition rate is pivotal for the future progress of ultra-high intensity science and its potential real-world applications. Here we present the conceptual design of a diodepumped cryogenic kJ-class Yb:YAG amplifier as a potential pump source for multi-PW Ti:Sapphire or OPCPA systems.

\subsubsection{Choice of gain medium}

As there is currently no gain material available that allows high-energy, ultra short $(<100 \mathrm{fs})$ pulse generation from a directly diode pumped laser, the best approach is to use a ns-pulse DPSSL for pumping large-aperture OPCPA or Ti:Sapphire amplifiers. The gain material for such a pump laser requires a long fluorescence lifetime in order to minimise the number of pump diodes required; good thermo-mechanical properties in order to handle the high average power; it must also be available in large sizes and good optical quality in order to handle the high pulse energy; and it should provide a reasonably high gain cross section to enable simple and efficient energy extraction.

$\mathrm{Yb}: \mathrm{YAG}$, in particular in ceramic form, fulfils all these requirements. However, at room temperature it exhibits a quasi3-level nature and a still rather low gain cross section, necessitating very high fluence levels for efficient operation. The Boltzmann occupancy factor for the Stark sub-level of the $2 \mathrm{~F} 7 / 2$ manifold for the peak transition at $1030 \mathrm{~nm}\left(612 \mathrm{~cm}^{-1}\right)$ is $4.6 \%$ at room temperature, reducing to $0.64 \%$ at $175 \mathrm{~K}$. This reduction in lower laser level population leads to a more four-level-like behaviour with greatly reduced reabsorption losses. Also, low temperature operation leads to increased adsorption and gain cross sections and improved thermo-mechanical properties. The reduction in gain bandwidth at low temperature is no major drawback in this context.

\subsubsection{Amplifier design and modelling}

In order to determine amplifier design parameters, numerical modelling was carried out. In this model, the extractable storage efficiency was calculated for various parameters like pump fluence and pump pulse duration. The following results are calculated, unless stated otherwise, for an amplifier that is end-pumped from both sides with pump pulse 
duration of $1 \mathrm{~ms}$, a $5 \mathrm{~nm}$ FWHM pump spectral width, centred at the optimum wavelength in the $940 \mathrm{~nm}$ absorption band. Spectrally resolved pump absorption cross sections were taken from [22]. First, calculations were carried out for room temperature operation shown by the square data points in Fig. 7. It becomes apparent that very strong pumping is required, firstly to overcome the high reabsorption losses and to achieve good efficiency and secondly to overcome the still rather low gain cross section to achieve reasonable gain. The required high pump and extraction fluences are difficult to achieve because of limited pump source brightness and limited laser damage threshold.



Figure 7. Maximum storage efficiency (solid line with square) and small signal gain (dotted line with square) for amplifier operated at room temperature. Corresponding maximum storage efficiency (solid line with triangle) and small signal gain (dotted line with triangle) as a function of pump fluence at $175 \mathrm{~K}$ operation.

Cooling the gain medium to $175 \mathrm{~K}$ drastically changes the situation, as illustrated in Fig. 7 (triangles). Reabsorption is reduced and the gain cross section increased, leading to greatly improved efficiency and gain, especially at moderate fluences. A pump fluence that is realistically achievable with today's laser diodes is $10 \mathrm{~J} / \mathrm{cm}^{2}\left(5 \mathrm{~J} / \mathrm{cm}^{2}\right.$ from each side), yielding a storage efficiency of just over $50 \%$ (resulting in an extractable fluence of $5 \mathrm{~J} / \mathrm{cm}^{2}$ ) and a small signal gain of 3.8. This fluence is therefore chosen as the preliminary operating point for our amplifier.

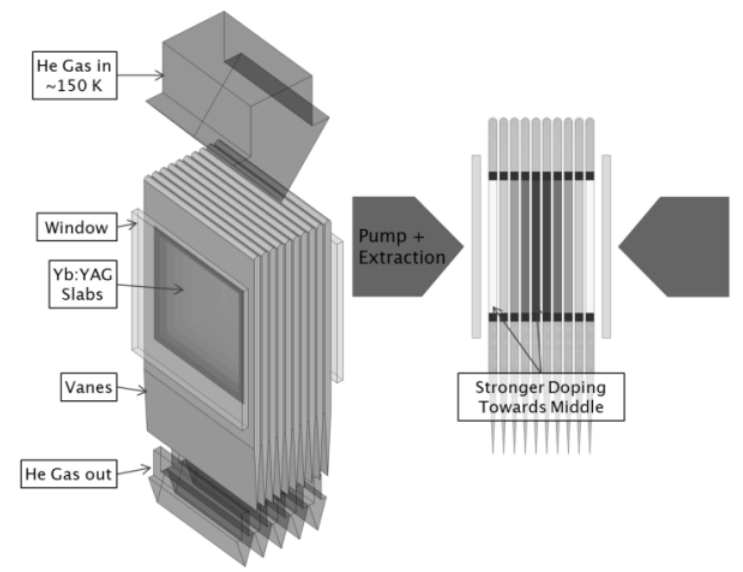

Figure 8. Illustration of amplifier geometry: isometric (left) and side view (right).

After determining operating temperature and pump fluence, the actual geometry of the amplifier needs to be defined. If the laser system is to yield an output energy of $1 \mathrm{~kJ}$ and the amplifier is to be operated at an output fluence of $5 \mathrm{~J} / \mathrm{cm}^{2}$, the aperture needs to be $200 \mathrm{~cm}^{2}$ or $14 \times 14 \mathrm{~cm}^{2}$ if a square beam shape is adopted. The optimum area doping density Nxl obtained from the numerical calculations is $3.15 \% \mathrm{~cm}$, where $\mathrm{N}$ is the $\mathrm{Yb}$-doping concentration in atomic $\%$ and 1 the geometrical thickness of the amplifier. The choice of $\mathrm{N}$ and consequently 1 is governed by ASE management considerations. If the gain-length product along the diagonal across the (square) surface of the amplifier is to be kept below 3, we require $\mathrm{N}<0.18 \%$ and hence $1>18 \mathrm{~cm}$. Such a thick amplifier requires distributed cooling, such as demonstrated on the Mercury laser [23]. There, the gain medium is divided into a stack of thin slabs with He gas flowing through the gaps in between. The concept is illustrated in Fig. 8, where an amplifier consisting of 10 slabs is shown. If the criterion that the transverse gain-length product must not exceed a certain value is applied to each individual slab, 
one realises that the doping concentration can be increased towards the centre of the amplifier. The advantage is twofold: firstly, since the required overall $\mathrm{Nxl}$ remains the same, the amplifier as a whole becomes thinner, facilitating pumping, saving material, and reducing the impact of nonlinear effects. Secondly the optical power absorbed in the individual slabs and hence the heat load is equalised.

\section{BASELINE CONCEPT OF THE ELI LASERS}

Baseline scheme of the ELI research infrastructure, as resulted from works and analysis carried out thus far within the ELI-Preparatory Phase project, is shown in Fig 9. The ELI will consist of four main sections. Attosecond section, Beamline applications section, Nuclear physics section and Extreme physics section. The individual sections will provide different combinations of pulse energy, repetition rate, peak and average power.

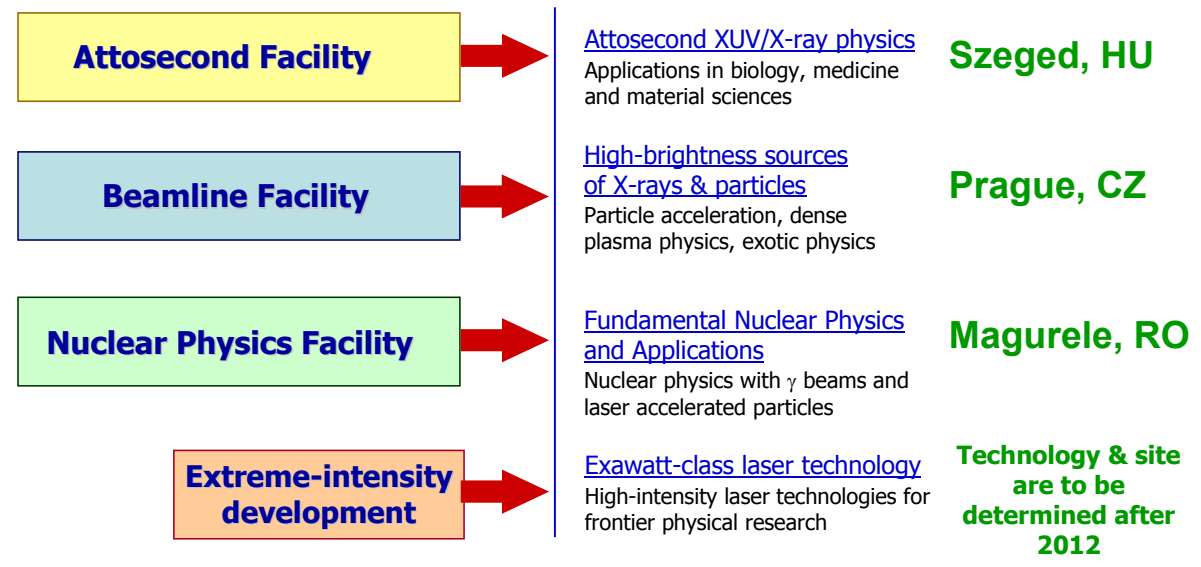

Figure 9. Concept of the ELI research infrastructure.

The attosecond section will be based on the PFS technique and will constitute a single (or several) petawatt-class lasers running at typically $\mathrm{kHz}$ repetition rate.

At least some ELI beamlines must run at $10-\mathrm{Hz}$ repetition rate, if ELI is to become a competitive source of accelerated electrons or protons on the world scale. Feeding target areas for electron and ion acceleration, the pulse length should be flexible since generation of e.g. $10 \mathrm{GeV}$ electrons will require 50 to $100 \mathrm{fs}$ pulses. Energy of the pulses delivered by the beamline should be at least $50 \mathrm{~J}$ (after compression). It appears that at least two beamlines should be dedicated to electron and protons acceleration. Due to large bandwidth, the OPCPA technique will be employed as furthest down the beamlines as possible.

The extreme-intensity section will deliver a sum peak power of at least $200 \mathrm{PW}$, with nominal repetition rate smaller than that of the beamlines $(<<0.1 \mathrm{~Hz})$. As design options, systems including flashlamp pumping of Ti:Sapphire, of OPCPA, and of combination of both are to be explored.

\subsection{ELI Attosecond Science Facility, Szeged, Hungary}

The ELI Attosecond Facility will be built in Szeged, about $165 \mathrm{~km}$ south-east from Budapest. Szeged is the capital of the region hosting the largest university of Hungary outside Budapest - with about 25 thousand students and 6 thousand employees - as well as the Biological Research Center of Hungary. The building construction is to start in late 2011, while the first target area is expected to become operational in early 2015.

The primary mission of the Hungarian ELI Facility is to provide the scientific community with short wavelength light sources, especially with coherent XUV radiation, including single attosecond pulses and attosecond pulse trains. The driving laser is a PW class, few cycle system with an unprecedented repetition rate of $1 \mathrm{kHz}$. All the known methods of attosecond pulse generation will be utilized. The well-established high harmonic generation in rare gases will be pushed 
to its ultimate limits, aiming at producing attosecond pulses with energies on the ten $\mu \mathrm{J}$ scale. Much more energetic and shorter as pulses can be generated on surfaces of solids [24]. This method is not yet fully explored, so research will also be directed for the full exploitation of the technique. Finally, the use of external fields as multi colour light radiation or even DC electric field, has been recently proposed to enhance the generation process of as pulses. For this purpose $\mathrm{THz}$ pulses are indepedently generated and their use for as generation will be implemented.

The secondary purpose of the research infrastructure is to contribute to the scientfic and technological development towards the extreme intensity facility (see Section 3.4). For this purpose a second laser system, similar to APOLLON, is planned at a peak power of around $20 \mathrm{PW}$ at a moderate repetition rate of 1 shot / minute. Besides the study of different amplification schemes, the issue of coherent beam combination will be primarily investigated with superposition of the laser pulses from the two branches.

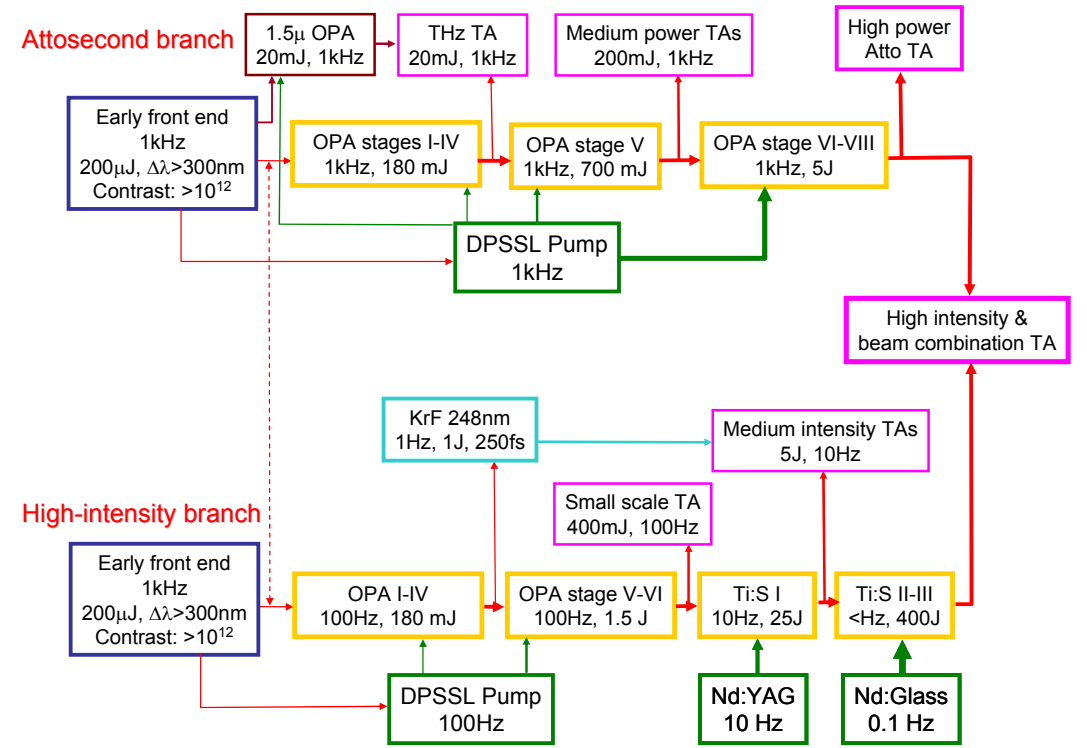

Figure 10. Current schematics of the laser and target areas of the ELI Attosecond Facility. For synchronised operation of the two branches, only one of the early front end feeds both laser chains, as indicated by the dashed line.

The two laser branches will have identical early front ends, consisting of a commercial laser system working at $800 \mathrm{~nm}$ and with $1 \mathrm{kHz}$ repetition rate (Fig. 10). The DPSSL pump laser at $1.03 \mu \mathrm{m}$ as well as the $1.5 \mu \mathrm{m}$ satellite amplifier for $\mathrm{THz}$ generation will be fed by parts of the $5 \mathrm{~mJ}$, sub-30 fs laser pulses. The seed pulses for both the above $900 \mathrm{~nm}$ (atto-) branch and the $800 \mathrm{~nm}$ (high-intensity) branch will be generated from the largest part of the commercial system through spectral broadening and subsequent XPW filtering, so that few cycle, high temporal contrast laser pulses enter the OPCPA stages. While the repetition rate and bandwidth of the pulses in the atto-branch are expected to be kept at $1 \mathrm{kHz}$ and $>300 \mathrm{~nm}$, respectively, the final amplifiers of the high intensity system will be based on flashlamp pumped Ti:S technology, providing higher pulse energies but limited to a lower repetition rate

The research infrastructure will operate as a facility for internal and external users. As it is, efforts will be devoted to provide the users with all kind of light pulses of the host lasers as well as the generated secondary sources as HHG, as and $\mathrm{THz}$ pulses, but also their unique combinations. We hope that ELI-Hu will then significantly contribute to basic researches in physics, chemistry, bio-sciences and pharmacology, as well as to material sciences and techniques in medical diagnostics and therapy.

\subsection{ELI Beamline Facility, Prague, Czech Republic}

The schematic layout of the laser system of the ELI-Beamlines facility is shown in Figure 11. The front end will consist of the master Ti:Sapphire oscillator providing $>300 \mathrm{~nm}$ equivalent bandwidth pulses, and an array of preamplifiers delivering 8 mutually synchronized and appropriately timed pulses at $\sim 10 \mathrm{~mJ}$ level. These pulses will be pulses into booster rep-rate amplifiers based on the PFS technique and pumped by thin disk lasers currently prototyped at MPQ in Garching and MBI in Berlin. The system will involve two types of the booster amplifiers: the first type running at $1 \mathrm{kHz}$ 
and providing equivalent energy of $200 \mathrm{~mJ}$ in the compressed pulse, the second type running at $100 \mathrm{~Hz}$ and delivering equivalent energy of about $1 \mathrm{~J}$. The first type will deliver pulses directly to the experimental areas, whereas the second type will feed the beamline power amplifiers and the 10-PW test laser blocks.

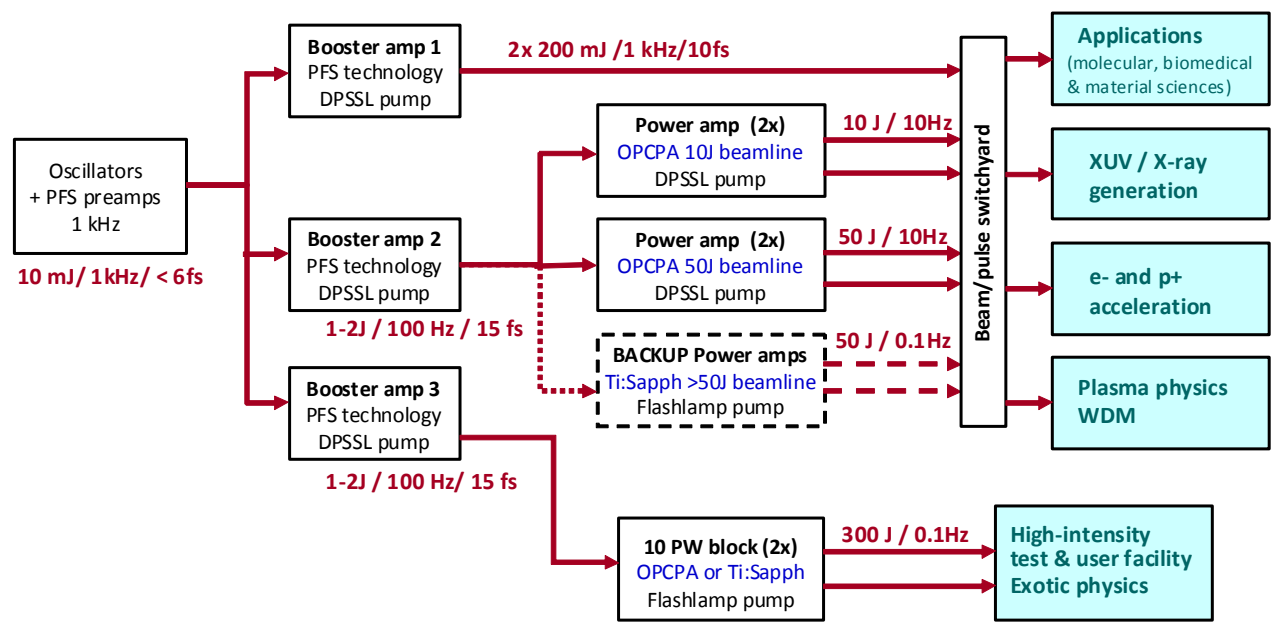

Figure 11: Block layout scheme of the ELI-Beamlines laser facility (pulse compressors are not represented).

The beamline power amplifiers will be based on the OPCPA technique, driven by rep rate DPSSL system multi-slab cryogenic amplifiers, running at a frequency of $10 \mathrm{~Hz}$. The facility will involve two types of the DPSSL-pumped beamlines. The first type will deliver approximately $10 \mathrm{~J}$ of compressed energy, implying $\sim 100 \mathrm{~J}$ (at the fundamental wavelength of $1.03 \mu \mathrm{m}$ ) of pump energy. The second type will provide about $50 \mathrm{~J}$ of compressed energy and will require $\sim 500 \mathrm{~J}$ pump pulses. The fallback solution for the latter type is Ti:Sapphire technology using as pump systems conventional Nd:YAG flashlamp lasers (or, alternatively, Nd-based heat capacity lasers). This technology is matured and commercially available. If used here, the beamlines will provide repetition rate of $0.1-1 \mathrm{~Hz}$. The 10-PW laser system will provide compressed pulses with energy of 200-300 J and pulse duration of 20-30 fs, with repetition rate of approximately $0.1 \mathrm{~Hz}$. Two technology options which will be prototyped in near future (10 PW Vulcan and Apollon) are considered: OPCPA and Ti:Sapphire.

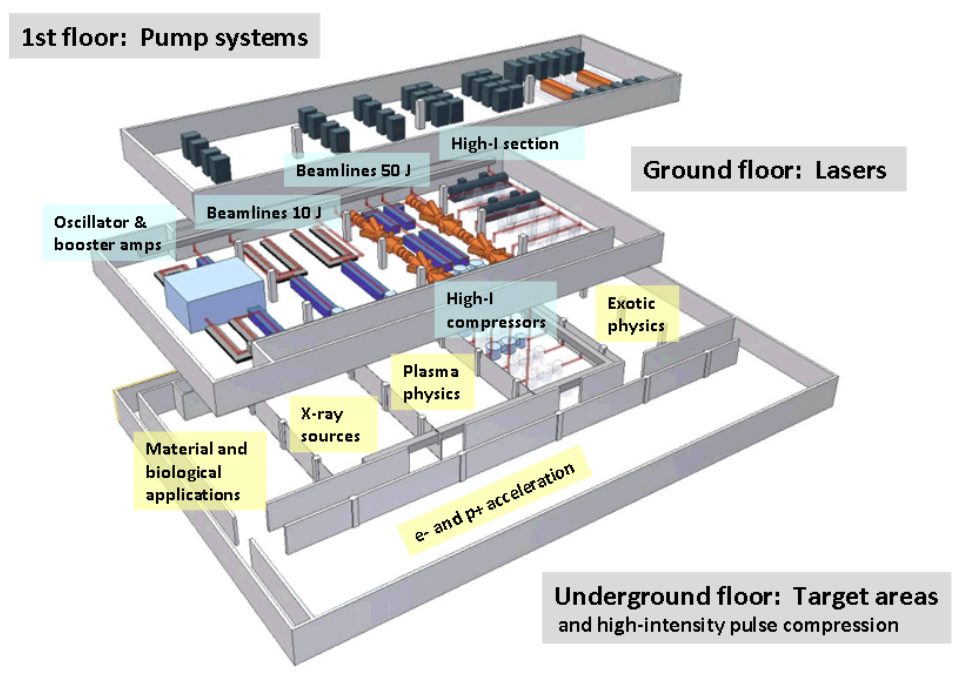

Figure 12: Layout of the ELI-Beamlines facility. The laser systems, including oscillator \& front end, repetition rate beamlines and a high-intensity test system are located in the ground floor, the driving and pump systems are placed in the 1st floor. The underground floor consists of experimental areas and of a pulse compression room.

Layout of the designed ELI-Beamlines facility laser is shown in Figure 12. The individual laser systems are located in the ground floor of a monolithic structure ensuring high level of mechanical and thermal stability. The laser area is divided into four subsections, hosting the front end a booster amplifiers, 10-J repetition rate beamlines, 50-J repetition 
rate beamlines, and high-intensity amplifiers. Overhead the laser systems in the first elevated floor are pump and supporting systems (drivers of diode lasers, flashlamp pump lasers, capacitor banks, cryogenic systems, etc.) The final compressors of the 10-PW laser pulses are located below the amplifiers in the underground floor which provides flexibility of delivery of the large-aperture high-intensity beams into the experimental areas.

\subsection{ELI Nuclear Physics Facility, Bucharest, Romania}

The facility to be placed in Magurele, near Bucharest, in Romania, will mainly focus on laser-based nuclear physics. The ELI Nuclear Physics (ELI-NP) facility will generate $\gamma$ and particle beams with high energies and brilliances suited to studies of nuclear and fundamental processes. The core of the facility is a laser system using Ti:Sapphire technology. In order to perform cutting edge nuclear physics experiments, a complementary highly brilliant $\gamma$ beam with energies in the $15 \mathrm{MeV}$ range, will be generated via the laser interaction with a brillant bunched electron beam. Thus ELI-NP will allow either combined experiments between the high power laser and the $\gamma$ beam or PW laser stand-alone experiments. The design of the facility is modular, reserving the space for further extension of the laser system and allowing the extension of the experimental area at a later moment in time, according to the needs.

The ELI-NP laser facility architecture is depicted in Figure 13. It will use OPCPA technology at the front-end and Ti:Sapphire high-energy amplification stages, similar to the ones developed at the Apollon laser system described in the section 2.1. A front-end based exclusively on Ti:sapphire oscillator and amplifiers, with XPW for high intensity contrast, is considered as a back-up solution. The ELI-NP laser facility will have two front-ends. They will temporally stretch and amplify initial ultrashort pulses with $800 \mathrm{~nm}$ central wavelength to the $100 \mathrm{~mJ}$ level, preserving the needed large bandwidth of the $15 \mathrm{fs}$ laser pulses and the temporal contrast of the pulses in the range of $10^{-12}$. Due to the complexity of such OPCPA system, the allignment and maintenance time for one front-end is long. To avoid such dead-times, one front-end is planned to operate at a time, the second one being used during the maintenance of the other front-end, significantly increasing the available beam-time of the laser facility.

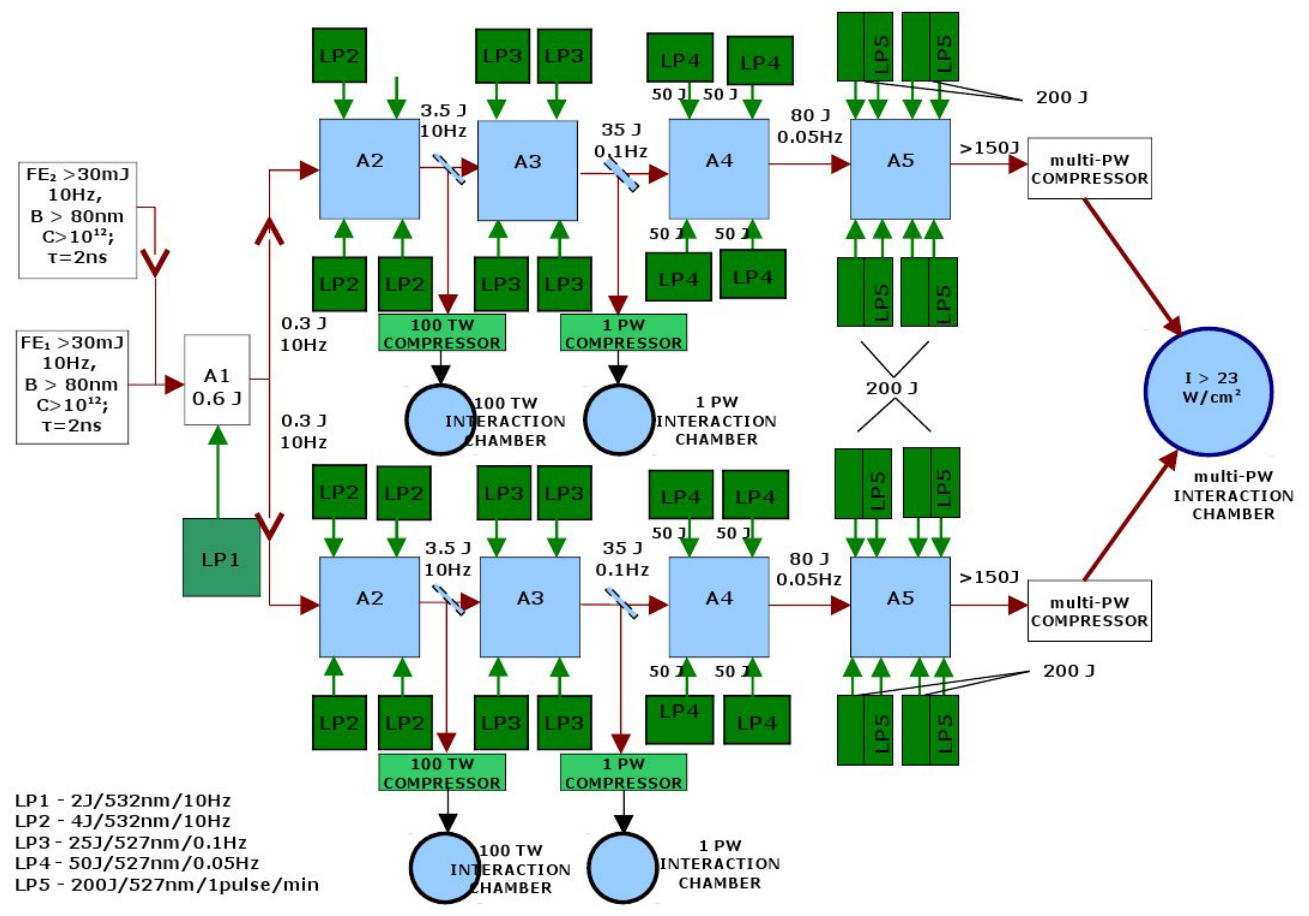

Figure 13: Architecture of the ELI-NP laser system. It combines OPCPA front-ends with Ti:Sapphire amplifiers.

The pulses after the front end are splitted and distributed to further laser amplifiers, reaching few Jules of energy at 10 $\mathrm{Hz}$ repetition rate and few tens of Joules at a repetition period of the order of few seconds. At these energy levels, the pulses can be extracted from the laser amplification chain and recompressed to shortest duration in vacuum compressors. Subsequently, they are distributed to the high repetition rate experimental areas. 
Alternatively, the laser pulses are further amplified in the amplification chains to energies of the order of $300 \mathrm{~J}$. The repetition rate of the pump lasers will restrict the repetition period of the high energy pulses to the minutes range. Adaptive optics and optical isolation of the pulses will be implemented before the optical mosaic compressors. The ultrashort pulses will be distributed to the high energy experimental areas, where stand-alone experiments or combined nuclear physics experiments using the highly brilliant $\gamma$ beam will be performed.

Coherent combination of the high power ultrashort pulses with the ultraintense and ultrashort pulses from the parallel amplification chains is envisaged, in order to reach intensities of the order of $10^{23} \mathrm{~W} \mathrm{~cm}^{-2}$ and above. The operation of the experiments will take place in parallel, the laser pulses being delivered to different experimental areas on request.

The experimental program of the ELI-NP facility addresses both fundamental science research and application-oriented developments. Fundamental physics of perturbative and non-perturbative high-field QED, high resolution nuclear spectroscopy and astrophysics-related studies of r-, s-, and p-processes in nucleosynthesis are included in the basic scientific research. The emerging applications are related to the development of the nuclear resonance fluorescence (NRF) reactions for radioactive waste management, to the use of brilliant $\gamma, \mathrm{X}, \mathrm{n}, \mathrm{e}^{+}, \mathrm{e}^{-}$microbeams in material science and life science and to the development of techniques of laser acceleration and of brilliant $\gamma$ beam for nuclear physics.

\subsection{ELI Extreme Intensity Pillar}

The design and construction of the exawatt-class laser system is the far largest challenge of the community. Major technological and scientific bottlenecks include not only those already associated with the planned laser facilities, but primarily centered around the problem of generation of $200 \mathrm{PW}$ peak power pulses on a target. There are two approaches. One is to generate such power in a single laser beam. This, however, would require optics, primarily diffraction gratings, laser crystals and nonlinear converters in about 4-6 times larger size than the aimed ones at the $10 \mathrm{PW}$ facilities. Since the latter are already challenging, this approach is unlikely to be successful within a few years.

The other approach aims at the combination of 8 to 12 laser beams with 10-20 PW power each. Combination of high intensity, high aperture laser beams have been already demonstrated for long (nanosecond) pulses at the National Ignition Facility (NIF) and Laser Mega Joule (LMJ), and are certainly planned in HiPER. At ELI, the difficulty arises from the pulse duration: it is very challenging to combine accurately laser beams where the pulse duration is less than 15 fs. Not only the intensity envelopes have to be combined with $<0.1 \mu \mathrm{m}$ accuracy, but also the electric fields have to overlap accurately in order to keep the carrier envelope phase uniform within the pulse and across the beam.

Besides of this fundamental question, the laser technology suitable at exawatt level has not been clarified either. Namely, the community wishes further investigate whether Ti:S based amplification, or optical parametric amplifiers would suit best to generate such an extreme power.

All the three ELI Facilities described above along with the already running or planned national programs like APOLLON, Gemini, PFS, VULCAN and DiPOLE, and will contribute to the research and development efforts to find solutions to the technological and scientific bottlenecks. Special attention is to be paid to find synergies with HiPER to solve common issues in laser technology and targetry. The scientific community hence suggested providing all the projects a few years time, and postponing the decisions, including the site of the exawatt facility, after 2012.

\section{SUMMARY}

ELI will be the first facility in the world dedicated to laser-matter interaction in the ultra-relativistic regime $\left(>10^{24} \mathrm{~W} / \mathrm{cm}^{2}\right)$, providing unprecedented intensity levels. It will be the gateway to new regimes in physics. At the same time, it will also promote new technologies such as relativistic microelectronics with the development of compact laseraccelerators delivering $>100 \mathrm{GeV}$ particles and photon sources. ELI will have a large societal benefit in new diagnostic techniques in medicine, in compact hadron therapy and PET applications, and in material sciences. The single governance Pan-European research infrastructure to be implemented in at least three sites will provide several types of laser pulses with different combination of the pulse energy, pulse duration, and repetition rate. The most intense ELI pulses will be delivered by coherent combination of several beams and will provide a total peak power $200 \mathrm{PW}$ or higher. 


\section{REFERENCES}

[1] www.eli-laser.eu

[2] G.Mourou, T. Tajima, S.V Bulanov, "Optics in the relativistic regime", Rev.Mod.Phys. 78, 309-371 (2006)

[3] E. Gertner, "Extreme Light", Nature 446, 16-18 (2007)

[4] M. Dunne, "A high power fusion laser facility for Europe", Nature Phys. 2, 2-5 (2006)

[5] Bor, Z., "Distortion of femtosecond laser pulses in lenses," Opt. Lett. 14, 119-121 (1989).

[6] Chekhlov, O., Divall, E. J., Ertel, K., Hawkes, S. J., Hooker, C. J. and Collier, J. L., "Beam switching and beam transport for the Astra Gemini project," Central Laser Facility Annual Report 2006/2007, 165-167 (2007).

[7] Ertel, K., Hooker, C., Hawkes, S. J., Parry, B. T. and Collier, J. L., "ASE suppression in a high energy Titanium sapphire amplifier," Opt. Express 16, 8039-8049 (2008).

[8] Kneip S., et al, "Near-GeV Acceleration of Electrons by a Nonlinear Plasma Wave Driven by a Self-Guided Laser Pulse," Phys. Rev. Letters 103, 035002 (2009).

[9] C. Wandt, S. Klingebiel, M. Siebold, Zs. Major, J. Hein, F. Krausz, and S. Karsch, "Generation of $220 \mathrm{~mJ}$ nanosecond pulses at a $10 \mathrm{~Hz}$ repetition rate with excellent beam quality in a diode-pumped Yb:YAG MOPA system", Opt. Lett. 33, 1111 (2008)..

[10]M. Siebold, J. Hein, C. Wandt, S. Klingebiel, F. Krausz, and S. Karsch , "High-energy, diode-pumped, nanosecond Yb:YAG MOPA system", Optics Express, 16, 3675 (2008).

[11] I. Ahmad, S. A. Trushin, Zs. Major, C. Wandt, S. Klingebiel, T.-J. Wang, V. Pervak, A. Popp, M. Siebold, F. Krausz, S. Karsch, "Frontend light source for short-pulse pumped OPCPA system", Appl. Phys. B 97, 529 (2009)

[12] V. Pervak, I. Ahmad, S. A. Trushin, Zs. Major, A. Apolonski, S. Karsch, and F. Krausz, "Chirped-pulse amplification of laser pulses with dispersive mirrors", Opt. Exp. 17, 19204 (2009).

[13]Zs. Major, S. Trushin, I. Ahmad, M. Siebold, C. Wandt, S. Klingebiel, T.-J. Wang, J. A. Fülöp, A. Henig, S. Kruber, R. Weingartner, A. Popp, J. Osterhoff, R. Hörlein, J. Hein, V. Pervak, A. Apolonski, F. Krausz, and S. Karsch, "Basic concepts and current status of the Petawatt Field Synthesizer - a new approach to ultrahigh field generation", Review of Laser Engineering 37, 431 (2009).

[14] M. Siebold, M. Loeser, U. Schramm, J. Koerner, M. Wolf, M. Hellwing, J. Hein and K. Ertel, "High-efficiency, room-temperature nanosecond Yb:YAG laser", Opt. Exp. 17, 19888 (2009).

[15] J. A. Fülöp, Zs. Major, A. Henig, S. Kruber, R. Weingartner, T. Clausnitzer, E.-B. Kley, A. T"unnermann, V. Pervak, A. Apolonski, J. Osterhoff, R. Hörlein, F. Krausz and S. Karsch, "Short-pulse optical parametric chirped-pulse amplification for the generation of high-power fewcycle pulses", New J. Phys. 9, 438 (2007).

[16] I. N. Ross et al, Opt. Commun. 144, 125-133 (1997)

[17] I.N.Ross，J.L.Collier，P.Matousek，C.N.Danson，D.Neely，R.M.Allott，D.A.Pepler，C.Hernandez-Gomez, K.Osvay, "The generation of terawatt pulses using optical parametric chirped pulse amplification", Appl.Opt. 39, 2422-2427 (2000)

[18] J. Collier, C. Hernandez-Gomez, I.N. Ross, P. Matousek, C. Danson, J. Walczak, "Evaluation of an ultrabroadband highgain amplification technique for chirped pulse amplification" Appl. Opt. 38, 7486 (1999)

[19] I.N.Ross, P.Matousek, G.H.C.New, K.Osvay, "An Analysis and Optimisation of Optical Parametric Chirped Pulse Amplification", J.Opt.Soc.Am. B 19, 2945-2956 (2002)

[20] J.L.Collier et al, IFSA 2003, American Nuclear Society, Pub: Elsevier, 603-607, (2004)

[21] Y Tang et al, Opt.Lett. 33, 2386 (2008)

[22]D. C. Brown, IEEE J Sel Top Quant. 11, 587-599 (2005)

[23] A. Bayramian, P. Armstrong, E. Ault, R. Beach, C. Bibeau, J. Caird, R. Campbell, B. Chai, J. Dawson, C. Ebbers, A.Erlandson, Y. Fei, B. Freitas, R. Kent, Z. Liao, T. Ladran, J. Menapace, B. Molander, S. Payne, N. Peterson, M.Randles, K. Schaffers, S. Sutton, J. Tassano, S. Telford, E. Utterback, Fusion Sci Technol 52, 383387 (2007)

[24] G. Tsakiris, K. Eidmann, J. Meyer-ter-Vehn, F. Krausz, "Route to intense single attosecond pulses", New J. Phys. 8, 19 (2006) 